\title{
Procrastinar academicamente é coisa de perfeccionista? Correlatos valorativos e da personalidade
}

\author{
¿Procrastinar académicamente es una cuestión perfeccionista? \\ Correlación entre el valor y la personalidad \\ Procrastinating ficademically is Something of a Perfectionist? \\ Ualue and Personality Correlations
}

\author{
Ana Karla Silva Soares \\ Universidade Federal do Mato Grosso do Sul \\ Daniely Fernandes Kamazaki \\ Universidade Federal do Rio Grande do Sul \\ Sandra Elisa de Assis Freire \\ Universidade Federal do Piauí - Campus Parnaíba
}

Doi: https://doi.org/10.12804/revistas.urosario.edu.co/apl/a.8687

\section{Resumo}

O presente estudo objetivou conhecer o poder preditivo dos traços de personalidade, dos valores humanos e do perfeccionismo para com a procrastinação acadêmica, propondo um modelo explicativo. Participaram 348 universitários, a maioria do sexo feminino (58.4\%), com idade média de 22 anos (DP = 5.9). Estes, responderam a Escala de Procrastinação Acadêmica; a Escala de Quase Perfeição - Revisada; o Questionário dos Valores Básicos; o Inventário dos Cinco Grandes
Fatores da Personalidade e perguntas demográficas. Baseado na regressão, propôs-se um modelo explicativo no qual a conscienciosidade predisse as dimensões de perfeccionismo (adaptativo e desadaptativo) e estas, por sua vez, predisseram a procrastinação acadêmica. Os resultados apontam para um ajuste satisfatório deste modelo que contribuem para o entendimento da procrastinação acadêmica.

Palavras-chave: procrastinação; perfeccionismo; valores; testes de personalidade; universitários.

\footnotetext{
Ana Karla Silva Soares ORCID ID: https://orcid.org/0000-0001-5306-4073

Daniely Fernandes Kamazaki ORCID ID: https://orcid.org/0000-0002-1556-7451

Sandra Elisa de Assis Freire ORCID ID: https://orcid.org/0000-0003-1083-6963

Dirigir correspondência à Ana Karla Silva Soares. Endereço: av. Costa e Silva - Pioneiros, MS, CEP: 79070-900, Brasil.

Correio eletrônico: akssoares@gmail.com
}

Para citar este artigo: Soares, A. K. S., Kamazaki, D. F., \& Freire, S. E. A. (2021). Procrastinar academicamente é coisa de perfeccionista? Correlatos valorativos e da personalidade. Avances en Psicología Latinoamericana, 39(1), 1-16. https://doi.org/10.12804/ revistas.urosario.edu.co/apl/a.8687 


\section{Resumen}

Este estudio tuvo como objetivo comprender el poder predictivo de los rasgos de personalidad, los valores humanos y el perfeccionismo hacia la procrastinación académica, proponiendo un modelo explicativo. Participaron 348 estudiantes universitarios, la mayoría mujeres (58.4\%), con una edad media de 22 años $(\mathrm{DE}=5.9)$. Estas respondieron a la Escala de Procrastinación Académica; la Escala de Casi Perfección - Revisada; el Cuestionario de Valores Básicos; el Inventario de los Cinco Grandes Factores de la Personalidad y preguntas demográficas. A partir de un análisis de regresión, se propuso un modelo explicativo en el que la escrupulosidad predijo las dimensiones del perfeccionismo (adaptativo y desadaptativo) y estas, a su vez, predijeron la procrastinación académica. Los resultados apuntan a un ajuste satisfactorio de este modelo que contribuye a la comprensión de la procrastinación académica. Palabras clave: procrastinación; perfeccionismo; valores; pruebas de personalidad; estudiantes universitarios.

\section{fibstract}

The present study aimed at discerning the predictive power of personality traits, human values, and perfectionism towards academic procrastination, proposing an explanatory model. Participants were 348 undergraduates, most of them female (58.4\%), with a mean age of 22 years $(S D=5.9)$. They answered the Academic Procrastination Scale, the Almost Perfect Scale - Revised, the Basic Values Survey, the Big Five Inventory, and demographic questions. Based on regression analysis, an explanatory model was proposed where conscientiousness predicted the dimensions of perfectionism (adaptive and maladaptive) that in turn predicted academic procrastination. Results suggested a satisfactory fit of the model to the indices that contributes to the understanding of academic procrastination.

Keywords: Procrastination; perfectionism; values; personality tests; undergraduates.
Algum dia você já deixou de fazer algo mesmo sabendo que adiar para mais tarde poderia piorar as coisas por conta do atraso? Ou ainda realiza suas atividades com muito zelo buscando que tudo seja perfeito? Se respondeu sim a alguma destas questões ou as duas, seja bem-vindo ao grupo dos chamados "procrastinadores" e/ou "perfeccionistas", especialmente se for (ou foi em algum dia) estudante universitário.

As pessoas em geral tendem a procrastinar a realização de algumas atividades e compromissos em suas vidas. Estes comportamentos refletem diretamente na maneira como cada pessoa vivencia seu dia a dia, gerando sensações de nível elevado de estresse, raiva, arrependimento, pânico e insatisfação com as atividades realizadas devido à falta de tempo para execução, não sendo rara tanto entre a população geral, cerca de $20 \%$ (Ferrari et al., 2007) quanto entre estudantes universitários, com pelo menos $50 \%$ dos acadêmicos (Closson \& Boutilier, 2017), relatando dificuldades em concluir suas atividades curriculares e aspectos que englobam motivação e desempenho acadêmico (Bong et al., 2014; Kim et al., 2017).

$\mathrm{Na}$ literatura, identificam-se estudos que conectam o comportamento de procrastinação a dimensões subjacentes, que afetam o contexto universitário e outras áreas da vida, estando mais associada a emoções negativas, sentimentos de incerteza, ansiedade, medo do fracasso e, consequentemente, preocupações perfeccionistas (Kurtovic et al., 2019; Sirois et al., 2017). O perfeccionismo é definido dentro da estrutura adotada por Slaney et al. (2001) como também tendo aspectos positivos e negativos, consistindo na tendência a ser excessivamente focado nas imperfeições, erros, críticas e discrepâncias percebidas entre realizações reais e ideais.

Apesar da relação entre o perfeccionismo e a procrastinação ser consistentemente corroborada na literatura, com estudos relacionando-os a aspectos mais resistentes à mudança como critérios 
de orientação (valores humanos) (Grund \& Fries, 2018; Stoeber \& Yang, 2016) ou características da personalidade humana (Kim et al., 2017; Naami \& Salehi, 2016; Stricker et al., 2019), são escassos os estudos que se dedicam a avaliar a correlação destas variáveis em conjunto. Tendo em conta os aspectos supracitados, considera-se oportuno discutir de maneira pormenorizada a procrastinação, o perfeccionismo, os valores humanos e a personalidade, o que se faz a seguir.

\section{Procrastinação e Perfeccionismo}

Considera-se que a procrastinação e o perfeccionismo estão relacionados em virtude do medo excessivo que alguns indivíduos possuem do fracasso, e, que padrões elevados de perfeccionismo podem promover a procrastinação (Shim et al., 2016), visto que os perfeccionistas tendem a temer comparações com seus pares, sendo o adiamento das tarefas uma maneira de fugir da avaliação e de uma possível falha ou classificação como "incompetente". Por outro lado, pessoas podem procrastinar para evitar críticas dos outros, usando desculpas para não iniciar suas atividades. Assim, a procrastinação tem sido definida como a prática de adiar a realização de atividades, podendo ser de forma deliberada ou não (Kim et al., 2017).

Ferrari et al. (1995) definiram procrastinação como um padrão de comportamento com atrasos frequentes no início e/ou na conclusão de tarefas até o prazo final, sendo extremamente comum e relacionado a sintomas como ansiedade e estresse. Dessa forma, compreende-se a procrastinação como um atraso voluntário de uma ação e, embora possua uma intenção de realizar a ação, há uma expectativa de uma consequência negativa resultante do atraso. Kurtovic et al. (2019) ressaltam que embora haja a procrastinação em diferentes áreas, esta é mais comumente associada ao contexto educacional e organizacional, visto os prazos externos e específicos estabelecidos para a realização de uma tarefa ou um trabalho.
O interesse sobre o efeito da procrastinação no contexto acadêmico não é atual, com pesquisadores como Solomon e Rothblum (1984) relacionando-a com a demora em concluir deveres de casa, preparar-se para exames ou finalizar trabalhos no último instante da data de entrega, com uma prevalência média de $50 \%$ em estudantes da época, percentual mantido e até ampliado por pesquisas mais atuais como na de Ferrari e Ferrari (2010) que identificou $70 \%$ dos acadêmicos com algum nível de procrastinação, e no estudo de Argiropoulou et al. (2016) na Grécia, que identificou cerca de $40.5 \%$ dos alunos classificados como procrastinadores frequentes.

Diante do previamente exposto, uma variável que se associa a procrastinação é o perfeccionismo, definido como a determinação de padrões extremamente elevados, voltados à procura pela perfeição (Smith et al., 2016). Logo, se caracteriza pela busca de excelência e pela tendência em estabelecer padrões elevados de desempenho, acompanhada pela tendência de autoavaliação hipercrítica e uma crescente preocupação em cometer erros (Stoeber et al., 2011).

O perfeccionismo abrange o estabelecimento de padrões extremamente elevados, direcionados a busca pela perfeição geralmente definido como uma dimensão multidimensional que abrange aspectos mais saudáveis/insalubres ou adaptativos/desadaptativos (Closson \& Boutilier, 2017). Na medida elaborada por Slaney et al. (1996) identificam-se três fatores que são distribuídos em duas dimensões:

a. Perfeccionismo adaptativo. É constituído pela reunião de dois fatores: (1) padrões elevados, medem o nível de altas expectativas que alguém tem para si mesmo e (2) ordem, que avalia a preferência de uma pessoa por ordem e limpeza. Indivíduos que pontuam alto neste tipo de perfeccionismo, tendem, a estabelecer e manter padrões individuais elevados, mas apresentam a capacidade de se orgulhar de seus feitos e praticar a autoaceitação quando não atingem seus excessivos objetivos (Moate et al., 2019). 
b. Perfeccionismo desadaptativo. É caracterizado pelo fator discrepância, definido como a diferença percebida entre os próprios padrões e o desempenho real, sendo característico de indivíduos que estabelecem altos padrões pessoais, mas apresentam a vivência de forte autocrítica quando são incapazes de satisfazer seus elevados padrões (Arana et al., 2017; Moate et al., 2019). $\mathrm{Na}$ tentativa de capturar a complexidade do perfeccionismo, identificam-se definições mais abrangentes, que enfocam comportamentos e cognições autodirigidas, bem como expectativas dos pais (Hewitt \& Flett, 1991) ou componentes conceitualmente definidos a partir da Teoria dos Cinco Grandes Fatores da Personalidade (McCrae \& John, 1992) pautados nos traços de concienciosidade e neuroticismo denominados de Perfeccionismo Consciente (focado na busca por organização, esforço para excelência, planejamento e altos padrões para outros) e Perfeccionismo Auto-Avaliador (direcionado a preocupação com erros, necessidade de aprovação, ruminação e pressão parental percebida) (Hill et al., 2004).

Dessa forma, segundo Kurtovic e colaboradores (2019), ao considerar a relação entre o perfeccionismo e a procrastinação, os pesquisadores indicam que muitas pessoas procrastinam porque são perfeccionistas, já que os perfeccionistas tendem a estabelecer padrões irrealistas para si e procrastinam por acreditar que esses padrões não podem ser atingidos. Os perfeccionistas tendem a recorrer a comportamentos característicos para alcançar seus objetivos que são controlar e monitorar todos os aspectos relacionados à conformidade ou manter os padrões perfeccionistas. Tais ações podem ser condutas ativas ou passivas como a procrastinação, sendo então o perfeccionismo um possível precursor da procrastinação (Flett et al., 2004). Diante destes construtos, é necessário que seja possível encontrar aspectos psicológicos que possam predizê-los. Nesse sentido, os valores humanos e a personalidade nos ajudam a compreender essa relação.

\section{Valores Humanos e Traços de Personalidade}

O termo valores é popularmente conhecido e empregado pelas pessoas no cotidiano, principalmente ao tratar de temáticas que envolvam aspectos morais e éticos. No contexto científico há diversas teorias e definições de valores humanos, (Inglehart, 1990; Rockeach, 1973; Schwartz, 1994) e nesta pesquisa opta-se por empregar uma teoria elaborada no contexto brasileiro (Gouveia, 2013), com comprovação de adequação em mais de 50 países (Soares, 2015) e que se apresenta mais parcimoniosa e integradora que as demais, denominada Teoria Funcionalista dos Valores Humanos (Gouveia et al., 2014).

Para Gouveia (2013), os valores são compreendidos como princípios ou guias desejáveis e representação cognitiva das necessidades, tendo por objetivo assegurar a sobrevivência e evolução da sociedade. O principal foco da teoria são as subfunções valorativas, tendo como funções primárias: o tipo de orientação e o tipo de motivador. A primeira refere-se à função dos valores como guia dos comportamentos formados por três tipos de orientação: social (o indivíduo na comunidade), pessoal (o indivíduo por si mesmo) e central (o propósito geral da vida). A segunda diz respeito à função dos valores de expressar cognitivamente as necessidades, sendo divididas em dois tipos: materialista (pragmática) ou idealista (humanitária).

Assim, o cruzamento das duas dimensões valorativas tipo de orientação (horizontal) e tipo de motivador (vertical) geram uma estrutura $3 \times 2$ composta por seis subfunções, distribuídas entre os critérios de orientação social (interativa e normativa), central (suprapessoal e existência) e pessoal (experimentação e realização). Assim como, entre os tipos motivadores idealista (interativa, suprapessoal e experimentação) e materialista (normativa, existência e realização).

As pessoas que adotam os valores pessoais procuram alcançar metas pessoais, garantia de 
benefícios próprios e condições propícias para o alcance de seus objetivos, sendo caracterizado pelas subfunções: experimentação (emoção, prazer e sexualidade), cujos valores favorecem a promoção de mudança e inovação na estrutura das organizações sociais e realização (êxito, poder e prestígio) na qual as pessoas focalizam realizações materiais e buscam a praticidade em decisões e comportamentos.

Os indivíduos compatíveis com os valores sociais priorizam a convivência social e se relacionam com os interesses coletivos e com a busca por aceitação e integração ao grupo, sendo subdivididos com base nas subfunções: normativa (obediência, religiosidade e tradição), pessoas direcionadas a respeitar as regras sociais e preservar a cultura e as normas sociais; e interativa (afetividade, apoio social e convivência) que remete às necessidades de pertença, amor e afiliação, promovendo o estabelecimento e a manutenção das relações interpessoais por parte do indivíduo.

Enquanto que as pessoas que priorizam valores centrais são aquelas que servem a interesses mistos e localizam-se entre os valores pessoais e sociais formados pelas subfunções: existência (estabilidade pessoal, saúde e sobrevivência) que se relaciona com as condições fisiológicas mais básicas (beber, dormir, comer) e a necessidade de segurança, principalmente a de sobrevivência biológica e psicológica e suprapessoais (beleza, conhecimento e maturidade) que representam as necessidades estéticas, de cognição e de autorrealização.

Assim, os valores humanos evidenciam-se como importantes variáveis preditoras de diferentes temáticas, incluindo a procrastinação e o perfeccionismo. Grund e Fries (2018) investigaram a relação entre orientações valorativas de realização, bem-estar (Teoria de Hofer e Fries) e procrastinação a um nível geral, identificando que os alunos com maior preferência por valores de realização e que preferiram menores indicadores de bem-estar, apresentaram menor tendência a procrastinar, sugerindo que a procrastinação reflete um descompasso entre o engajamento atual e as estruturas motivacionais básicas. Enquanto Flett et al. (1995) identificaram que a dimensão de perfeccionismo relacionada a padrões pessoais (ser organizado e ordenado) se relacionou positivamente com o compromisso de se comportar de uma maneira moral e ética.

Ao considerar a relevância dos valores, faz-se necessário ampliar o núcleo preditivo ao inserir a personalidade como outro importante construto, sendo utilizado para explicar atitudes, comportamento e também valores humanos. Parks-Leduc et al. (2015) estudaram por meio de uma metanálise a relação entre os cinco grandes fatores da personalidade e os valores humanos, corroborando o papel preditivo da personalidade e sua forte relação com as prioridades valorativas dos indivíduos.

$\mathrm{Na}$ literatura, são identificados diferentes teóricos da personalidade como B. F. Skinner, Sigmund Freud, Abraham Maslow, Carl Rogers e aqueles que priorizaram o estudo dos traços de personalidade, a exemplo de Gordon Allport, Raymond Cattell e Hans Eysenk. Por fim, será dada ênfase aos chamados Cinco Grandes Fatores da Personalidade (BIG 5), uma vez que, no que concerne à personalidade, é nessa abordagem que o presente estudo está baseado, segundo o qual cada fator é nomeado a partir de um traço geral que abarca características e semânticas sobre cinco dimensões básicas: extroversão, neuroticismo, socialização (amabilidade), realização (conscienciosidade) e abertura à mudança, ressaltando a possibilidade de variação quanto a terminologia utilizada (Silva \& Nakano, 2011). Deste modo, observa-se um consenso entre os pesquisadores quanto aos cinco grandes fatores, visto que eles constituem um modelo integrativo que possui poder satisfatório de sintetizar os aspectos que compõem a personalidade humana (Araújo, 2013), a saber:

- Abertura à mudança - Também chamado de Cultura, Imaginação ou Intelecto. As pessoas que pontuam alto nesse fator são consideradas criativas, curiosas, com flexibilidade nos pensamentos, abertura a mudanças, são adeptas 
de novas ideias, valores não-convencionais e interesses culturais e artísticos.

- Conscienciosidade - Chamado também de Falta de impulsividade, realização ou Vontade. Esse fator é característico de indivíduos com estabilidade motivacional para alcançar objetivos e controle de impulsos. Logo, pontuações altas nesse fator indicam pessoas organizadas, persistentes, responsáveis e cautelosos. Por isso, indivíduos conscienciosos geralmente são dignos de confiança.

- Extroversão - Também chamado de Expansão. Esse fator relaciona-se aos modos como as pessoas interagem com as demais. Sujeitos que pontuam alto nessa escala tendem a ser sociáveis, ativos, falantes, otimistas, afetuosos, comunicativos, entusiasmados, dominantes, comportando-se costumeiramente, com liberdade e desenvoltura.

- Amabilidade - Chamado também de Agradabilidade ou Sociabilidade. Esse fator avalia o nível de empatia dos sujeitos e o quão prestativos são com os demais, isto é, a qualidade de suas relações interpessoais. Nesse sentido, pessoas com escores altos refletem uma tendência para a estabilidade social, tendendo a terem um padrão comportamental pró-social. Elas geralmente são generosas, bondosas, afáveis, prestativas, altruístas, agradáveis, amáveis, cooperativas e afetuosas.

- Neuroticismo - Chamado também de Instabilidade Emocional. Assim, indivíduos neuróticos geralmente apresentam instabilidade emocional e são mais propensos a sofrimentos psicológicos, demonstrando ideias irreais, baixa tolerância à frustração e respostas de enfrentamento não adaptativas. Os indivíduos são nervosos, altamente sensíveis, tensos, inseguros e preocupados.

Kennedy e Tuckman (2013) analisaram a relação entre os valores sociais e a procrastinação, com os resultados sugerindo que a procrastinação pode ser mediada parcialmente pela influência dos valores acadêmicos e sociais na percepção de pertença dos estudantes à escola. Neste seguimento, Kim et al. (2017), examinaram a relação entre a procrastinação e os cinco traços de personalidade em universitários, com seus achados identificaram relação entre a procrastinação e a extroversão e o neuroticismo.

Considerando os achados relacionais com o perfeccionismo, historicamente, autores da personalidade clássicos como o Alfred Adler (1938), consideraram que os perfeccionistas estão "comparando-se perpetuamente com a ideia inalcançável de perfeição, são sempre possuídos e estimulados por um sentimento de inferioridade" (pp. 35-36). Smith et al. (2018) discute sobre os esforços perfeccionistas caracterizarem-se pela dimensão de conscienciosidade e quando orientado para outros é caracterizado principalmente pela baixa amabilidade.

Adicionalmente, observa-se que alguns autores tratam o perfeccionismo como um traço da personalidade (Frost et al., 1990; Hewitt \& Flett, 1991) e outros analisam sua relação com os diferentes traços (Naami \& Salehi, 2016; Stoeber \& Corr, 2017), cujos resultados variam em detrimento da medida/definição empregada. Identificam correlação com o traço neuroticismo $(+)$, conscienciosidade $(+$ e -), extroversão (-) e amabilidade (-) (Hill et al., 2004; Steel \& Klingsieck, 2016).

Uma busca recente nas bases de dados Scientific Electronic Library Online (scielo, 2019) e Periódicos Eletrônicos de Psicologia (PePSIC, 2019), tendo por expressões-chave "perfeccionismo" AND "procrastinação" $A N D$ "valores" $A N D$ "personalidade", não identificou nenhum estudo relacionando as quatro variáveis no contexto brasileiro.

Nesta direção, partindo dos pressupostos teóricos identificados na literatura, vislumbrou-se a realização do presente estudo, tendo em conta dois objetivos principais: conhecer o poder preditivo dos traços de personalidade, dos valores humanos e do perfeccionismo para com a procrastinação acadêmica; e propor um modelo explicativo de preditores da procrastinação acadêmica. 


\section{Método}

\section{Participantes}

Contou-se com uma amostra de conveniência (não probabilística) de 348 estudantes universitários, sendo a maioria de universidades públicas (92\%), do sexo feminino ( $58.4 \%$ ), que não trabalha $(69.1 \%)$, com idade média de 22 anos (variando de 18 a 54 anos; DP = 5.9). Quando indagados sobre o quanto se consideram bons estudantes, $50 \%$ se classificaram como bons alunos.

\section{Instrumentos}

Escala de Procrastinação Acadêmica. Originalmente elaborada em idioma inglês por McCloskey (2011), a medida é constituída por 25 itens onde os participantes são convidados a indicar o nível de concordância com as afirmações utilizando uma escala do Likert, variando de 1 (concordo) a 5 (discordo). A versão original identificou estrutura unifatorial, evidência de precisão $(\alpha=0.96)$ e validade convergente com outras medidas de procrastinação (e.g. Escala de Tuckman), resultados semelhantes ao identificado nesta pesquisa (unifatorial; $\alpha=0.78$; correlação com Escala de Tuckman, $r=0.80$ ). Para esta pesquisa identificou-se valores de precisão satisfatórios $(\omega=0.80 ; \alpha=0.76)$.

Escala de quase perfeição - revisada (Almost Perfect Scale - Revised; APS-R). A medida foi elaborada originalmente em língua inglesa por Slaney et al. (1996), sendo constituída por 23 itens distribuídas por três subescalas: padrões elevados (7 itens), discrepância (12 itens) e ordem (4 itens), que reunidas avaliam duas dimensões: perfeccionismo adaptativo (padrões elevados + ordem) e desadaptativo (discrepância) respondidos em uma escala de 7 pontos variando de 1 (Discordo totalmente) a 7 (Fortemente de acordo). Slaney et al. (2001) relataram consistência interna satisfatória variando de 0.85 a 0.92 ; ademais identificaram correlações positivas com outras medidas de per- feccionismo que suportam sua validade concorrente (Ashby \& Rice, 2002). Para esta pesquisa identificaram-se valores de precisão satisfatórios nos fatores de perfeccionismo adaptativo $(\omega=0.72$; $\alpha=0.71)$ e perfeccionismo desadaptativo $(\omega=0.84$; $\alpha=0.83$ ).

Questionário dos Valores Básicos (QVB). Elaborada por Gouveia (1998; 2013), consta de 18 itens ou valores específicos, apresentando-se para cada um dos itens dois descritores que procuram representar seus conteúdos. Estes valores são distribuídos equitativamente em seis subfunções valorativas previamente descritas: experimentação, realização, existência, suprapessoal, interativa e normativa. Com o fim de respondê-los, o participante deve ler cada um com atenção e avaliar sua importância como um princípio-guia na sua vida, utilizando escala de sete pontos, variando de 1 (Totalmente não importante) a 7 (Totalmente importante). Soares (2015) identificou evidência de adequação psicométrica (validade e precisão) da medida tanto no contexto brasileiro, quanto em mais 50 países. Nesta pesquisa, o modelo hexafatorial apresentou indicadores globais de ajuste que corroboram a adequação da estrutura $\left(\chi^{2} / \mathrm{gl}=2.69 ; \mathrm{CFI}=0.91\right.$; RMSEA $=0.070$ (IC: 0.061-0.079).

Inventário dos Cinco Grandes Fatores da Personalidade (ICGFP). Originalmente elaborada por John e Srivastava (1999), será empregada uma versão reduzida constituída por 20 itens (Gouveia et al., no prelo) respondidos em uma escala de cinco pontos, variando de 1 (Discordo Totalmente) a 5 (Concordo Totalmente). Os itens são agrupados nos cinco fatores de personalidade: extroversão, amabilidade, conscienciosidade, neuroticismo e abertura à mudança. Gouveia et al. (no prelo) identificaram indicadores de consistência interna (alfa de Cronbach) variando de 0.56 a 0.72 . Para esta pesquisa identificou-se valores de precisão satisfatórios nos fatores de abertura à mudança $(\omega=0.80 ; \alpha=0.78)$, conscienciosidade $(\omega=0.69 ; \alpha=0.68)$, extroversão $(\omega=0.81 ; \alpha=0.79)$, amabilidade $(\omega=0.68 ; \alpha=0.68)$ e neuroticismo $(\omega=0.79 ; \alpha=0.77)$. 


\section{Procedimento}

Os dados foram coletados por meio de um questionário on-line (LimeSurvey) divulgado nas redes sociais (e.g., Facebook e Twitter). Todos os participantes se deparavam na página inicial da pesquisa com o Termo de Consentimento Livre e Esclarecido (TCLE, incluindo via para o participante), contendo informações sobre o caráter anônimo e voluntário da participação que obteve aprovação do Comitê de Ética em Pesquisa (CAAE: 79972517.8.0000.0021), sendo possível declinar a qualquer momento sem que isso acarretasse algum ônus para os voluntários. Em média, os participantes demoravam 20 minutos para responder os instrumentos.

\section{Análise de Dados}

Foram empregados para tabulação e análise de dados o pacote estatístico PASW e AMOS (versão 21). Especificamente, com o primeiro, foram calculadas estatísticas descritivas (média, desvio-padrão), além de análises de correlação de $r$ de Pearson, teste $t$ de Student e análise de regressão. Já com o segundo, foi realizada a path analysis, procurando testar o modelo de predição da procrastinação acadêmica partindo das variáveis que indicarem poder preditivo. Na ocasião, considerou-se a matriz de covariância, adotando o estimador Maximum Likelihood (ML) e os seguintes indicadores: $\chi^{2} / \mathrm{gl}$ (recomendável valores entre 2 e 3); Goodness-of-Fit Index - GFI, Comparative Fit Index - CFI, e Tucker-Lewis Index - TLI (recomendando-se valor próximo a 0.90 como indicador de ajuste do modelo) e Standardized Root Mean Square Residual - SRMR (valores inferiores a 0.10 , e de preferência inferior a 0.08 são considerados um bom ajuste) (Byrne, 2010; Tabachnick \& Fidell, 2013).

\section{Resultados}

Inicialmente, descrevem-se as pontuações da população estudada nos traços de personalidade, prioridades valorativas e níveis de procrastinação e perfeccionismo, contemplando achados da amostra total e separando quanto a variável sexo. Tais resultados podem ser vistos na tabela 1 .

Tabela 1

Análise descritiva de procrastinação, perfeccionismo, valores humanos e personalidade

\begin{tabular}{lcccccc} 
& \multirow{2}{*}{ Variáveis } & \multicolumn{2}{c}{ Amostra Total } & \multicolumn{2}{c}{ Meminino } \\
\cline { 2 - 7 } & Média & $\mathrm{dp}$ & Média & $\mathrm{dp}$ & Média & $\mathrm{dp}$ \\
\hline Procrastinação acadêmica & 2.83 & 0.70 & 2.86 & 0.64 & 2.80 & 0.74 \\
\hline Perfeccionismo & & & & & & \\
\hline Padrões elevados & 5.18 & 0.94 & 5.09 & 0.92 & 5.24 & 0.95 \\
\hline Ordem & 5.19 & 1.08 & 5.03 & 1.02 & 5.29 & 1.11 \\
\hline Discrepância & 4.46 & 1.15 & 4.20 & 1.11 & 4.63 & 1.15 \\
\hline Valores & & & & & & \\
\hline Experimentação & 5.05 & 1.13 & 5.11 & 1.14 & 5.01 & 1.12 \\
\hline Suprapessoal & 5.57 & 1.02 & 5.57 & 0.80 & 5.57 & 0.80 \\
\hline Interativa & 5.39 & 1.02 & 5.22 & 1.09 & 5.50 & 0.94 \\
\hline Realização & 4.72 & 1.07 & 4.80 & 1.11 & 4.67 & 1.04 \\
\hline Existência & 5.97 & 0.83 & 5.98 & 0.80 & 5.97 & 0.85 \\
\hline
\end{tabular}




\begin{tabular}{|c|c|c|c|c|c|c|}
\hline \multirow{2}{*}{ Variáveis } & \multicolumn{2}{|c|}{ Amostra Total } & \multicolumn{2}{|c|}{ Masculino } & \multicolumn{2}{|c|}{ Feminino } \\
\hline & Média & $\mathrm{dp}$ & Média & $\mathrm{dp}$ & Média & $\mathrm{dp}$ \\
\hline Normativa & 4.62 & 1.34 & 4.41 & 1.36 & 4.76 & 1.32 \\
\hline \multicolumn{7}{|l|}{ Personalidade } \\
\hline Abertura à mudança & 3.65 & 0.88 & 3.78 & 0.85 & 3.56 & 0.89 \\
\hline Conscienciosidade & 3.84 & 0.71 & 3.81 & 0.69 & 3.86 & 0.72 \\
\hline Extroversão & 3.39 & 1.00 & 3.34 & 1.02 & 3.42 & 1.00 \\
\hline Amabilidade & 3.97 & 0.74 & 3.87 & 0.72 & 4.03 & 0.74 \\
\hline Neuroticismo & 3.59 & 1.02 & 3.34 & 1.08 & 3.76 & 0.94 \\
\hline
\end{tabular}

Os resultados identificaram que existe diferença estatisticamente significativa entre os fatores de perfeccionismo adaptativo $[t(346)=-2.16$, $p<0.05, d=0.23]$ e desadaptativo $[t(346)=-3.44$, $p<0.01, d=0.38]$, as subfunções interativa $[t(346)=-2.57, p<0.01, d=0.28]$ e normativa $[t(346)=-2.37, p<0.05, d=0.26]$, os traços de personalidade amabilidade $[t(346)=-1.99$, $p<0.05, d=0.22]$ e neuroticismo [ $t(346)=-3.83$, $p<0.001, d=0.42]$ com as mulheres pontuando mais que os homens em todos estes fatores, diferentemente do traço abertura à mudança $[t(346)$ $=-2.30, p<0.05, d=0.25]$, em que os homens apresentaram maiores médias quando comparados às mulheres. Por fim, não foi identificada diferença estatisticamente significativa quanto à procrastinação acadêmica $(p>0.05)$.

Em seguida, com o fim de conhecer em que medida e direção as pontuações nos traços de personalidade, subfunções valorativas, fatores de perfeccionismo e procrastinação acadêmica se associaram, calcularam-se as correlações $r$ de Pearson. Como observado na tabela 2, os achados permitem verificar que a procrastinação acadêmica se correlaciona negativa e significativamente com os traços de personalidade conscienciosidade $(r=-0.37)$, extroversão $(r=-0.12)$ e positivamente com o neuroticismo $(r=0.12)$, enquanto que se relaciona negativamente com os valores das subfunções de existência $(r=-0.12)$ e normativos $(r=-0.18)$.
Ao correlacionar a procrastinação acadêmica ao perfeccionismo observa-se relação significativa e negativa com o fator perfeccionismo adaptativo $(r=-0.43)$. Ao passo que o perfeccionismo desadaptativo se correlacionou positiva e significativamente $(r=0.21)$ com a procrastinação acadêmica. Os demais padrões de relação entre as variáveis podem ser observados na tabela 2 .

Tomando por base tais resultados, decidiu-se verificar o poder preditivo dos traços de personalidade, das subfunções valorativas e das dimensões de perfeccionismo sobre a procrastinação acadêmica. Realizou-se uma análise de regressão linear múltipla com método enter, tomando-se como variável critério o fator geral de procrastinação acadêmica e como preditoras as variáveis que apresentaram correlação significativa com esta variável, especificamente, os traços de conscienciosidade, extroversão e neuroticismo, com as subfunções existência e normativa e com o perfeccionismo, sendo este último representado pelas dimensões de perfeccionismo adaptativo e desadaptativo.

De acordo com os achados, $25 \%$ da variabilidade da procrastinação acadêmica é explicada apenas por um traço de personalidade conscienciosidade e pelo perfeccionismo $[\mathrm{F}(7,339)=17.48$, $p<0.001]$, apresentando os seguintes pesos de regressão: conscienciosidade $(\beta=-0.13 ; p<0.05)$, perfeccionismo adaptativo $(\beta=-0.41 ; p<0.001)$ e perfeccionismo desadaptativo $(\beta=0.20 ; p<0.001)$. 


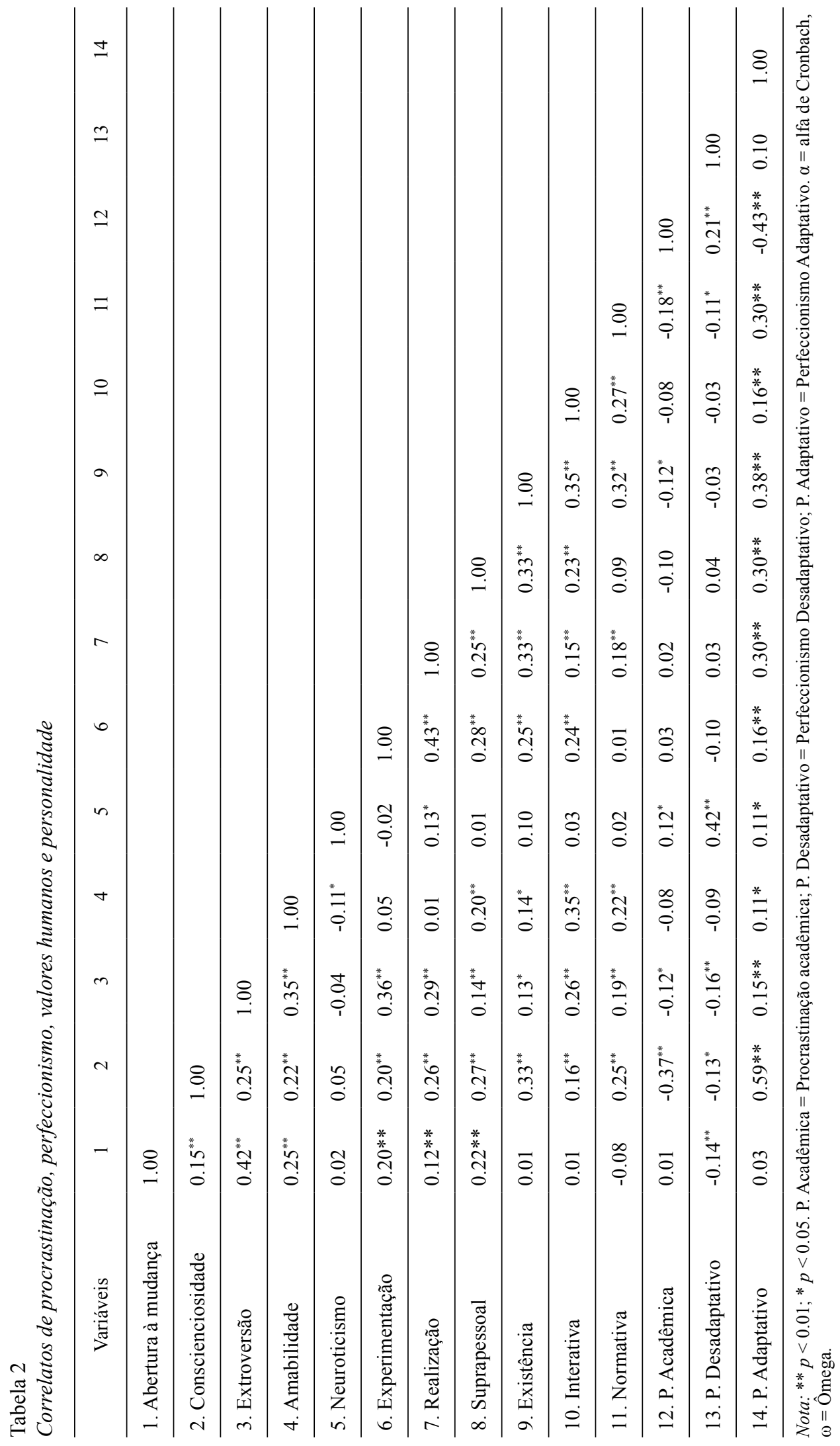


Tendo em consideração tais resultados, decidiu-se verificar a adequação de um modelo preditivo da procrastinação acadêmica, sendo testado por meio da modelagem por equações estruturais.

É possível observar que o modelo testado apresentou todos os lambdas estatisticamente significativos e diferentes de zero $(\mathrm{z}>1.96, p<0.05)$. Especificamente, como apresentado na figura 1, observa-se que o traço de personalidade conscienciosidade prediz positivamente o perfeccionismo adaptativo $(\lambda=0.59)$ e negativamente o perfeccionismo desadaptativo $(\lambda=-0.13)$, enquanto a procrastinação acadêmica é predita negativamente pelo perfeccionismo adaptativo $(\lambda=-0.45) \mathrm{e}$ positivamente pelo perfeccionismo desadaptativo $(\lambda=0.25)$. Além disso, os indicadores de ajuste desse modelo apresentam indicadores que, em conjunto, sugerem adequação do modelo, visto que apenas o TLI apresenta valor abaixo do recomendado $(>0.90)$ na literatura. $\mathrm{O}$ CFI que é considerado um índice menos afetado pela variabilidade da amostra (Bentler, 1990), foi satisfatório $(>0.90)$ : $\mathrm{GFI}=0.97, \mathrm{CFI}=0.93$, $\mathrm{TLI}=0.80 ; \mathrm{SRMR}=0.07$.

\section{Discussão}

Esta pesquisa buscou verificar o poder preditivo da personalidade, dos valores humanos e do per- feccionismo para com a procrastinação acadêmica e propor um modelo explicativo de preditores da procrastinação acadêmica. Diante dos resultados apresentados estima-se que os objetivos foram alcançados. Entretanto, os achados indicaram que apenas o traço de personalidade conscienciosidade e o perfeccionismo adaptativo e desadaptativo predizem a procrastinação acadêmica, sendo os mesmos empregados na formulação do modelo preditivo testado.

Foram identificadas diferenças significativas ao considerar a variável sexo e as pontuações dos participantes nos traços de personalidade amabilidade, neuroticismo e abertura à mudança, com os resultados sendo semelhantes ao reportado em pesquisas anteriores em que as mulheres pontuam mais alto que os homens em todos os fatores, com exceção da abertura à mudança (Costa et al., 2001).

Steel e Klingsieck (2016) verificaram que ao correlacionar os traços de personalidade e os níveis de procrastinação acadêmica em universitários, os resultados identificaram relação negativa para com a conscienciosidade e extroversão e relação positiva com neuroticismo. De modo semelhante, identificou-se nesta pesquisa o mesmo padrão de significância nas correlações. No caso do neuroticismo, se deu provavelmente em virtude do mesmo ser tido como uma forma de evitar contato com a ansiedade já que em tarefas acadêmicas importantes

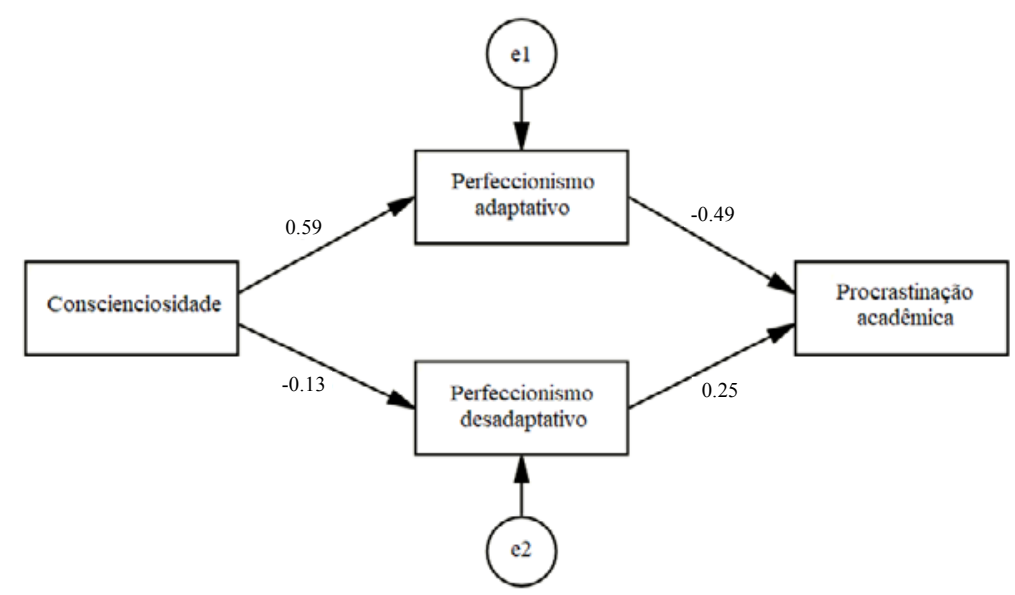

Figura 1. Modelo explicativo da procrastinação acadêmica 
(como estudar para provas e trabalhos) o estudante sente medo e ansiedade e tende a buscar atividades que o distraiam, uma vez que pessoas com traços de neuroticismo são mais vulneráveis a esses sentimentos e instáveis emocionalmente.

No que diz respeito ao traço de conscienciosidade dois aspectos importantes são destacados, a saber, a autodisciplina e a impulsividade. Uma vez que se apresentem características de estabilidade emocional, conseguindo controlar impulsos e se organizar em prol de alcançar objetivos, logo esta pessoa não procrastinará. Apenas o padrão correlacional para com extroversão difere do geralmente identificado na literatura (positivo; Kim et al., 2017) que concebe a dificuldade em autorregular o comportamento, como algo que levaria a tendência de procrastinar diante do impulso de adiar tarefas (principalmente tarefas consideradas difíceis ou chatas).

Por sua vez, a relação positiva observada entre perfeccionismo desadaptativo e a procrastinação acadêmica, indica que a percepção que as pessoas têm de que não estão cumprindo seus padrões elevados (Slaney et al., 1996), gera sentimento de culpa e arrependimento, prejudicando o bem-estar do estudante. Deste modo, considera-se que a relação entre procrastinação e o perfeccionismo resulta do medo excessivo em falhar, ou seja, com a preocupação em não cumprir os padrões (Shim et al., 2016).

A relação descrita anteriormente, auxilia na compreensão do resultado identificado em relação à extroversão daquele apontado pela literatura, já que Steel e Klingsieck (2016) sugerem que o traço de extroversão se relaciona com a procrastinação pela necessidade de socializar. Entretanto, considerando os achados do estudo, no que se refere à relação negativa entre o perfeccionismo desadaptativo e a extroversão, pode-se sugerir que ao passo em que se apresenta recorrentemente preocupações com a adoção de padrões elevados somada a ansiedade de traços neuróticos, mais dificuldades o estudante enfrentará para se socializar, devido ao foco dado às dificuldades acadêmicas, o que pode contribuir para ele não procrastinar.

Em relação aos valores humanos, a relação negativa entre procrastinação acadêmica e as subfunções normativa e existência, pode se dar pelo fato de que indivíduos que apresentam procrastinação acadêmica tendem a não priorizar as regras e normas sociais, nem preservar a cultura. Dessa forma, em contexto acadêmico, essas pessoas tendem a não seguir regras como prazos para trabalhos, nem priorizar as normas sociais impostas. E a existência apresenta como principal característica a busca com manutenção de necessidades básicas que envolvem busca por uma vida organizada e planejada seguindo padrões fixos, algo incompatível com comportamentos procrastinadores (Gouveia, 2013; Gouveia et al., 2014; Soares, 2015).

Entretanto, apesar das relações identificadas, ao proceder análises de natureza preditiva, apenas o traço de conscienciosidade apresentou poder preditivo da procrastinação acadêmica, acompanhado pelos dois tipos de perfeccionismo (adaptativo e desadaptativo). Portanto, os resultados do modelo teórico testado indicaram que é plausível pensar que pessoas conscienciosas que apresentam determinado padrão perfeccionista (adaptativo/ desadaptativo) explicam seus níveis de procrastinação acadêmica. Especificamente, pessoas mais conscienciosas tendem a ser mais adaptativamente perfeccionistas e menos procrastinadoras, enquanto as menos conscienciosas foram mais desadaptativamente perfeccionistas e mais procrastinadoras.

Contudo, embora os resultados identificados sejam relevantes para o entendimento destes construtos, faz-se necessário evidenciar potenciais limitações. Primeiramente, destaca-se o fato de a amostra utilizada ser de conveniência (não probabilística), ou seja, contou-se com a colaboração voluntária das pessoas abordadas para tal, sendo este aspecto o que impossibilita a generalização destes achados ao contexto de estudo. Outro aspecto envolve o emprego de medidas de autorrelato (tipo "lápis e papel”) que, apesar de serem as mais 
empregadas em estudos no geral, apresentam algumas desvantagens, a exemplo da possibilidade de os participantes falsearem intencionalmente suas respostas ou serem influenciadas pela desejabilidade social (Soares et al., 2016), que consiste na tendência das respostas se darem em virtude do que o respondente considera desejável pelo contexto social, especialmente ao considerar temáticas como perfeccionismo e procrastinação, comumente conceituadas como aspectos que afetam negativamente a saúde das pessoas.

Entretanto, estas limitações não invalidam os achados, que corroboram os pressupostos teóricos de que a personalidade é um fator importante na predição do perfeccionismo, considerado por vezes um traço da personalidade (Frost et al., 1990; Hewitt \& Flett, 1991; Stoeber et al., 2017) e que o perfeccionismo e a procrastinação estão associados, visto que comumente procrastina-se por ser perfeccionista, em virtude da busca pela perfeição envolver o estabelecimento de padrões individuais irreais e se procrastinar por considerar esses padrões inatingíveis (Closson \& Boutilier, 2017; Kurtovic et al., 2019; Shim et al., 2016). Futuramente, espera-se considerar outras formas de instrumental que permitam reduzir vieses de mensuração (e.g., medidas implícitas) e investigações mais aprofundadas sobre o funcionamento das mesmas em contextos culturais e públicos diferentes (e.g., população geral dos diferentes estados brasileiros).

\section{Referências}

Adler, A. (1938). Social interest: A challenge to mankind. Capricorn Books.

Arana, F. G., Rice, K. G., \& Ashby, J. S. (2017). Perfectionism in Argentina and the United States: Measurement structure, invariance, and implications for depression. Journal of Personality Assessment, 100(2), 219-230. https://doi.org/ $10.1080 / 00223891.2017 .1296845$
Araújo, R. D. C. R. (2013). As bases genéticas da personalidade, dos valores humanos e da preocupação com a honra (tese de doutorado). Departamento de Psicologia, Universidade Federal da Paraíba, João Pessoa, Brasil.

Argiropoulou, M. I., Sofianopoulou, A., \& Kalantzi-Azizi, A. (2016). The Relation Between General Procrastination and Health Behaviors: What Can We Learn from Greek Students? In F.M. Sirois \& T.A. Pychyl (Eds.), Procrastination, Health, and Well-Being (pp. 143-160). Elsevier.

Ashby, J. S., \& Rice, K. G. (2002). Perfectionism, dysfunctional attitudes, and self-esteem: A structural equations analysis. Journal of Counseling \& Development, 80(2), 197-203. https:// doi.org/10.1002/j.1556-6678.2002.tb00183.x

Byrne, B. M. (2010). Structural equation modeling with AMOS: basic concepts, applications, and programming (multivariate applications series). Taylor \& Francis Group.

Bong, M., Hwang, A., Noh, A., \& Kim, S. (2014). Perfectionism and motivation of adolescents in academic contexts. Journal of Educational Psychology, 106(3), 711-729. https://doi. org/10.1037/a0035836

Closson, L. M., \& Boutilier, R. R. (2017). Perfectionism, academic engagement, and procrastination among undergraduates: The moderating role of honors student status. Learning and Individual Differences, 57, 157-162. https://doi. org/10.1016/j.lindif.2017.04.010

Costa Jr, P. T., Terracciano, A., \& McCrae, R. R. (2001). Gender differences in personality traits across cultures: Robust and surprising findings. Journal of Personality and Social Psychology, 81(2), 322-331. https://doi.org/10.1037/00223514.81.2.322

Ferrari, J. R., Díaz-Morales, J. F., O'Callaghan, J., Díaz, K., \& Argumedo, D. (2007). Frequent behavioral delay tendencies by adults: International prevalence rates of chronic procrastination. Journal of Cross-Cultural Psychology, $38,458-464$. 
Ferrari, J. R., \& Ferrari, J. R. (2010). Still procrastinating: The no-regrets guide to getting it done. Wiley. Ferrari, J. R., Johnson, J. L., \& McCown, W. G. (1995). Procrastination research. In J.R. Ferrari, J.L. Johnson \& G. M. McCown (Eds.), Procrastination and Task Avoidance (pp. 21-46). Springer.

Flett, G. L., Hewitt, P. L., Davis, R. A., \& Sherry, S. B. (2004). Description and Counseling of the Perfectionistic Procrastinator. In H. C. Schouwenburg, C. H. Lay, T. A. Pychyl \& J. R. Ferrari (Eds.), Counseling the procrastinator in academic settings (pp. 181-194). American Psychological Association. https://doi.org/10.1037/10808-013

Flett, G. L., Hewitt, P. L., Blankstein, K. R., \& Mosher, S. W. (1995). Perfectionism, life events, and depressive symptoms: A test of a diathesis-stress model. Current Psychology, 14(2), 112-137. https://doi.org/10.1007/BF02686885

Frost, R. O., Marten, P., Lahart, C., \& Rosenblate, R. (1990). The dimensions of perfectionism. Cognitive Therapy and Research, 14(5), 449-468. https://doi.org/10.1007/BF01172967

Gouveia, V. V., Araújo, R. D. C. R., de Oliveira, I. C. V., Gonçalves, M. P., Milfont, T., de Holanda Coelho, G. L., ... \& Gouveia, R. (2021). A Short Version of the Big Five Inventory (BFI-20): Evidence on Construct Validity. Interamerican Journal of Psychology, 55(1), e1312-e1312. https://doi.org/10.30849/ripijp.v55i1.1312

Gouveia, V. V. (2013). Teoria funcionalista dos valores humanos: fundamentos, aplicações e perspectivas. Casa do Psicólogo.

Gouveia, V. V., Milfont, T. L., \& Guerra, V. M. (2014). Functional theory of human values: Testing its content and structure hypotheses. Personality and Individual Differences, 60(12), 41-47. https://doi.org/10.1016/j.paid.2013.12.012

Grund, A., \& Fries, S. (2018). Understanding procrastination: A motivational approach. Personality and Individual Differences, 121(1), 120-130. https://doi.org/10.1016/j.paid.2017.09.035

Hewitt, P. L., \& Flett, G. L. (1991). Dimensions of perfectionism in unipolar depression. Journal of Abnormal Psychology, 100(1), 98-101. https://doi.org/10.1037/0021-843X.100.1.98

Hill, R. W., Huelsmann, T. J., Furr, R. M., Kibler, J., Vicente, B. B., \& Kennedy, C. (2004). A new measure of perfectionism: The Perfectionism Inventory. Journal of Personality Assessment, 82(1), 80-91.https://doi.org/10.1207/s15327752jpa8201_13

Inglehart, R. (1991). Trust between Nations: Primordial Ties, Societal Learning and Economic Development. In K. Reif, \& R. Inglehart (Eds.), Eurobarometer. Palgrave Macmillan. https:// doi.org/10.1007/978-1-349-21476-1_9

Inglehart, R. (1990). Culture shift in advanced industrial society Princeton. Princeton. University Press.

John, O. P., \& Srivastava, S. (1999). The big-five trait taxonomy: History, measurement, and theoretical perspectives. In L. A. Pervin \& O. P. John (Eds.), Handbook of personality: Theory and research (Vol. 2, pp. 102-138). Guilford Press.

Kennedy, G. J., \& Tuckman, B. W. (2013). An exploration into the influence of academic and social values, procrastination, and perceived school belongingness on academic performance. Social Psychology of Education, 16(3), 435-470. https://doi.org/10.1007/s11218-013-9220-z

Kim, S., Fernandez, S., \& Terrier, L. (2017). Procrastination, personality traits, and academic performance: When active and passive procrastination tell a different story. Personality and Individual Differences, 108(1), 154-157.

Kurtovic, A., Vrdoljak, G., \& Idzanovic, A. (2019). Predicting Procrastination: The Role of Academic Achievement, Self-efficacy and Perfectionism. International Journal of Educational Psychology, 8(1), 1-26. https://doi.org/10.17583/ ijep.2019.2993

McCloskey, J. (2011). Finally, my thesis on academic procrastination (Master's thesis). University of Texas, Arlington.

McCrae, R. R., \& John, O. P. (1992). An introduction to the five-factor model and its applications. Jour- 
nal of Personality, 60(2), 175-215. https://doi. org/10.1111/j.1467-6494.1992.tb00970.x

Moate, R. M., Gnilka, P. B., West, E. M., \& Rice, K. G. (2019). Doctoral Student Perfectionism and Emotional Well-Being. Measurement and Evaluation in Counseling and Development, 13, 145-155. https://doi.org/10.1080/074817 56.2018 .1547619

Naami, A., \& Salehi, H. M. (2016). Prediction of Cosmetic Surgery Tendency Based on Mindfulness, Personality Dimensions, Perfectionism and Mental Health Components. International Journal of School Health, 3(3), 2-6. https://doi. org/10.17795/intjsh-32746.

Parks-Leduc, L., Feldman, G., \& Bardi, A. (2015). Personality traits and personal values: A meta-analysis. Personality and Social Psychology Review, 19(1), 3-29.

Rockeach, M. (1973). The nature of human values. Free Press.

Schwartz, S. H. (1994). Are there universal aspects in the structure and contents of human values? Journal of Social Issues, 50(4), 19-45. https:// doi.org/10.1111/j.1540-4560.1994.tb01196.x

Shim, S. S., Rubenstein, L. D., \& Drapeau, C. W. (2016). When perfectionism is coupled with low achievement: The effects on academic engagement and help seeking in middle school. Learning and Individual Differences, 45, 237 244. https://doi.org/10.1016/j.lindif.2015.12.016

Silva, I. B., \& Nakano, T. D. C. (2011). Modelo dos cinco grandes fatores da personalidade: análise de pesquisas. Avaliação Psicológica, 10(1), 51-62. https://dialnet.unirioja.es/descarga/articulo/6674920.pdf

Sirois, F. M., Molnar, D. S., \& Hirsch, J. K. (2017). A meta-analytic and conceptual update on the associations between procrastination and multidimensional perfectionism. European Journal of Personality, 31(2), 137-159. https://doi. org/10.1002/per.2098

Slaney, R. B., Mobley, M., Trippi, J., Ashby, J. S., \& Johnson, D. (1996). Almost Perfect Scale
Revised. Unpublished scale, Pennsylvania State University, University Park.

Slaney, R., Rice, K. G., Mobley, M., Trippi, J., \& Ashby, J. (2001). The Revised Almost Perfect Scale. Measurement and Evaluation in Counseling and Development, 34(3), 130-145.

Smith, M. M., Saklofske, D. H., Stoeber, J., \& Sherry, S. B. (2016). The Big Three Perfectionism Scale: A new measure of perfectionism. Journal of Psychoeducational Assessment, 34, 670-680.

Smith, M. M., Sherry, S. B., Vidovic, V., Saklofske, D. H., Stoeber, J., \& Benoit, A. (2018). Perfectionism and the five-factor model of personality: A meta-analytic review. Personality and Social Psychology Review, 1(1), 1-24. https:// doi. org/10.1177/1088868318814973.

Soares, A. K. S. (2015). Valores humanos no nível individual e cultural: Um estudo pautado na teoria funcionalista (tese de doutorado). Universidade Federal da Paraíba, João Pessoa, Brasil.

Soares, A. K. S., Lopes, G. S., Rezende, A. T., Ribeiro, M. G. C., dos Santos, W. S., \& Gouveia, V. V. (2016). Escala de Deseabilidad Social Infantil (EDSI): Evidencias de validez factorial y fiabilidad. Avances en Psicología Latinoamericana, 34(2), 383-394.

Solomon, L. J., \& Rothblum, E. D. (1984). Academic procrastination: Frequency and cognitive-behavioral correlates. Journal of Counseling Psychology, 31(4), 503. http://psycnet.apa.org/ fulltext/1985-07993-001.pdf

Steel, P., \& Klingsieck, K. B. (2016). Academic procrastination: Psychological antecedents revisited. Australian Psychologist, 51(1), 36-46.

Stoeber, J., \& Yang, H. (2016). Moral perfectionism and moral values, virtues, and judgments: Further investigations. Personality and Individual Differences, 88, 6-11. https://doi.org/10.1016/j. paid.2015.08.031

Stoeber, J., Childs, J. H., Hayward, J. A., \& Feast, A. R. (2011). Passion and motivation for studying: Predicting academic engagement and burnout in university students. Educational Psycho- 


\section{Ana Karla Silva Soares, Daniely fernandes Kamazaki, Sandra Elisa de fssis freire}

logy, 31(4), 513-528. https://doi.org/10.1080 $/ 01443410.2011 .570251$

Stoeber, J., \& Corr, P. J. (2017). Perfectionism, personality, and future-directed thinking: Further insights from revised Reinforcement Sensitivity Theory. Personality and Individual Differences, 105(5), 78-83. https://doi.org/ 10.1016/j.paid.2016.09.041

Stoeber, J., Noland, A. B., Mawenu, T. W., Henderson, T. M., \& Kent, D. N. (2017). Perfectionism, social disconnection, and interpersonal hostility: Not all perfectionists don't play nicely with others. Personality and Individual Differences, 119, 112-117. https://doi.org/10.1016/j. paid.2017.07.008

Stricker, J., Buecker, S., Schneider, M., \& Preckel, F. (2019). Multidimensional Perfectionism and the Big Five Personality Traits: A Meta-analysis. European Journal of Personality, 33(2), 176-196. https://doi.org/10.1002/per.2186

Tabachnick, B. G., \& Fidell, L. S. (2013). Using multivariate statistics (6th ed.). Pearson Education. 